International Journal of Advanced Trends in Computer Science and Engineering Available Online at http://www.warse.org/IJATCSE/static/pdf/file/ijatcse1151032021.pdf https://doi.org/10.30534/ijatcse/2021/1161032021

\title{
APSSR: Adaptive Packet Size Selection Based Routing Protocol for Underwater Acoustic Sensor Networks
}

\author{
Sajid Rehman Babar ${ }^{1}$, Waqas Saeed ${ }^{2}$, Hafiz M Mudassar Khan ${ }^{3}$, Akbar Ali $^{4}$ \\ Department of Computer Science, Sub Campus Gomal University Tank, Pakistan, srehmanbabar@ gmail.com \\ ${ }^{2}$ Department of Computer Science, Sub Campus Gomal University Tank, Pakistan, waqas.researchers@gmail.com \\ ${ }^{3}$ Department of Computer Science, Superior University, Lahore, Pakistan, hafizmudassarkhan @ gmail.com \\ ${ }^{4}$ Department of Computer Science, Federal University of Technology, Islamabad, Pakistan, aakbarali18@ gmail.com
}

\begin{abstract}
Underwater Acoustic Sensor Networks offer very promising solutions to monitor the aqueous environments. Due to the distinctive characteristics of UASNs, it is very challenging to design a routing protocol that can achieve maximum data delivery ratio in the network. The main challenge is the communication medium (acoustic links) that is subject to temporary attenuation and high bit error rate (BER), which limits the throughput efficiency of the Network. Besides this, another major issue is the continuous movement of nodes due to water currents and the availability of limited resources. Due to nodes mobility distance among sensor nodes and consequently, BER varies, which have a direct impact on packet size, hence, leads to high packet loss and low data delivery ratio. To achieve a high data delivery ratio, the selection of optimal packet size is of utmost importance. Consequently, the selection of next-hop forwarding node based on optimal packet size is needed. Therefore, in this paper, we propose an adaptive routing protocol named Adaptive Packet Size Selection Based Routing (APSSR) Protocol for UASNs. APSSR determines the optimal packet size adaptively based on both varying distances between sensor nodes and BER and selects the next hop based on optimal packet size and BER. The simulation results show greater network performance in terms of Network Lifetime, Data Reception Ratio at Sink node, Average Network Delay, Packet Reception Ratio, and Packets Drop Ratio.
\end{abstract}

Key words: Routing Protocol, Underwater Acoustic Communications, Optimal Packet Size, Physical Distance, BER, Packet Reception Ratio.

\section{INTRODUCTION}

The underwater acoustic sensor networks (UASNs) have gained a lot of interest in both academia and industry to assist an extensive range of underwater applications (i.e., Tsunami alerts, Undersea Exploration, Mine's detection, Pollution, and biological monitoring, etc.) [1]. Even though UASNs look like Terrestrial Wireless Sensor Networks (TWSNs), the radio waves used in TWSNs are not appropriate for UASNs [2]. The reason is that high-frequency radio waves face the problem of absorption due to water currents and noise, and low-frequency radio waves need long antennas and high transmission energy for communications [3]. As a result, only acoustic waves (sound waves) are used as a communication channel in UASNs [4]. However, the use of acoustic waves also includes many challenges in UASNs, such as attenuation, high propagation delays, low bandwidth, high energy

consumption, and high Bit Error Rate (BER) etc. [5]. The propagation speed of acoustic waves in UASNs is $1500 \mathrm{~m} / \mathrm{sec}$ and in case of TWSNs it is $3 \times 108 \mathrm{~m} / \mathrm{sec}$. Since the propagation delay (i.e. $0.67 \mathrm{sec} / \mathrm{km}$ ) in acoustic waves is five times higher than the electromagnetic waves [6]-[7], and acoustic waves offer very limited data rate (i.e. $40 \mathrm{~kb} / \mathrm{sec}$ at 1 $\mathrm{km}$ range) as compared to radio waves used in TWSNs. The bandwidth relies on distance and suffers from absorption factor of acoustic waves and ambient noises [8]. UASNs are considered as dynamic 3D networks due to nodes movement with water currents while traditional networks are mostly static 2D networks [9]. The energy consumed by nodes is higher in UASNs as compared to TWSNs because of their large size and the replacement of batteries is also not possible [10]. Due to low speed of acoustic waves, the links between nodes are highly error prone. Furthermore, in UASNs, acoustic communications suffer from high attenuation, and signal-to-noise ratio (SNR), etc. [11]. In UASNs, a group of sensor nodes are deployed randomly from top of the surface to bottom in their placement area, in order to sense, process, and transmit the data to destination node [12]. With all these constraints, designing a routing protocol which has the capability to achieve maximum throughput efficiency in this network is very challenging. Due to nodes mobility and low speed error prone acoustic communication medium, the rate of packet loss is usually high in this network. The reason is that distances among sensor nodes and BER varies and are not static. Therefore, the limited data delivery ratio is resulted. 
Various routing protocols for UASNs have already been proposed considering the unique characteristics of underwater environment [19], [20], [21], [27]. Although most of the schemes addressed the issue of forwarder node selection, none of them considers appropriate packet size selection. Most of the works has been done for link estimator algorithms, and the control packets are used for discovering link quality in UASNs. However, these control packets create additional

overhead in the network. Secondly, these protocols also use flooding to increase delivery ratio [13], [14], [25], [26], [27], [28]. However, due to flooding, energy consumption of nodes also increases which reduces the network lifetime. Thirdly, most of these protocols select the farthest forwarder node in its range. As the distance increases, bandwidth decreases as well as BER increases. It is observed that majority of routing protocols proposed for UASNs still do not consider the selection of appropriate (optimal) packet size based on both varying distances among sensor nodes and BER. Therefore, this paper takes into account both distance and BER information of sensor nodes and address the issue of inadequate packet size selection in UASNs, which influenced the data delivery ratio and overall network performance.

\subsection{Underwater Acoustic Sensor Network (UASN)}

\subsubsection{Acoustic Channel}

In UASNs, only acoustic waves are considered as the most reliable medium for communication. Since high frequency radio waves face the problem of absorption due to water currents, and low frequency radio waves need long antennas and high transmission energy for communication which increases network cost. Optical waves have short communication range and can only work up to $5 \mathrm{~m}$ in water. Therefore, optical waves cannot be used for long distance communications in distributed environment especially in deep water due to scattering problem. As a result, acoustic waves are only the reliable medium of communication for densely deployed, ad-hoc and low cost UASNs, since the frequency range of underwater acoustic signals is between $10 \mathrm{~Hz}$ and $1 \mathrm{MHz}$. Sound wave's propagation below $10 \mathrm{~Hz}$ is impossible without deep penetration in seabed while frequencies higher than $1 \mathrm{MHz}$ absorbed very quickly in water [13]. Despite of all benefits of acoustic waves as compared to other mediums (i.e., radio and optical waves) in water, acoustic signals also pose some challenges such as low bandwidth, long propagation delay, high path loss, high BER etc. Some of the factors affecting the acoustic channel are as follows:

\section{Path Loss}

Path loss occurs due to geometric spreading and signal attenuation. High frequency signals have absorption problem in underwater environment which causes attenuation. The absorption depends on distance. Therefore, the larger the distance is, the higher the absorption of the signal will be, and vice versa. The total amount of attenuation or path loss on basis of distance (d ) and signal frequency ( $\mathrm{f}$ ) is defined as follows [14]:

$A(d, f)=A_{g} d^{l} a(f)^{d}$

Where $\mathrm{d}=$ Distance $(\mathrm{m}), \mathrm{f}=$ Signal frequency, Ao $=$ Constant (i.e. unit normalizing constant), $\mathrm{k}=$ Geometry of propagation/Spreading factor (i.e. $1 \leq \mathrm{k} \leq 2)$, a(f) $=$ Absorption coefficient described by the Thorps formula in $(\mathrm{dB} / \mathrm{km})$

\section{Low-Bandwidth}

Low bandwidth results in low data rate. Bandwidth does not depend only on distance; it also depends upon the frequency of signals. Therefore, the systems that operate over very large distance can only get few hertz bandwidth. Although, acoustic communications is classified in different categories on the basis of its bandwidth and range, however, it is very difficult to exceed the data rate of $40 \mathrm{~kb} / \mathrm{sec}$ at $1 \mathrm{~km}$ range as shown in Table 1 [1].

\begin{tabular}{lcc}
\hline & Range $(\mathrm{Km})$ & Bandwidth $(\mathrm{KHz})$ \\
\hline Very long & 1000 & $<1$ \\
Long & $10-100$ & $2-5$ \\
Medium & $1-10$ & $\approx 10$ \\
Short & $0.1-1$ & $20-50$ \\
Very short & $<0.1$ & $>100$ \\
\hline
\end{tabular}

\section{Delay}

The propagation velocity in UASNs is $1500 \mathrm{~m} / \mathrm{sec}$ and in case of TWSNs, it is $3 \times 108 \mathrm{~m} / \mathrm{sec}$. Due to which the propagation delay (i.e. $0.67 \mathrm{sec} / \mathrm{km}$ ) in acoustic waves is five times higher than the electromagnetic waves. The velocity can be calculated through the empirical equation, if the values (i.e. temperature, salinity and depth) are obtained and is defined as follows [1]:

$\mathrm{v}=1449.2+4.6 \mathrm{~T}-0.055 \mathrm{~T}^{2}+0.00029 \mathrm{~T}^{2}+(1.34-0.010 \mathrm{~T})(5-35)+0.0162$

Where $\mathrm{v}$ is sound velocity $(\mathrm{m} / \mathrm{s}), \mathrm{T}$ is water temperature ${ }^{\circ} \mathrm{C}, \mathrm{S}$ is water salinity (parts per thousand (\%o)) and $\mathrm{z}$ is water depth (m). $0 \leq$ Temperature $\leq 35 \circ \mathrm{C}, 0 \leq$ Salinity $\leq 45 \%$, and $0 \leq$ depth $\leq 1000$ meter [28].

\section{Noise}

Ambient noises and man-made noises strongly affect acoustic communications in UASNs. Man-made noises are generated due to human activities such as ships while ambient noise is caused by fishes, wind, rain etc. [1]. The ambient noises can be modeled in the water through following sources: turbulence, shipping, wave and thermal noise. These 
four-noise components depend on frequency of the signal and can be computed using the below equations and is defined as follows [14]:

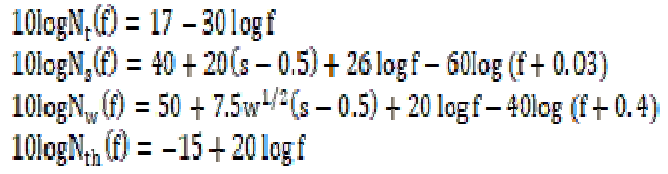

The total noise power spectral density (p.s.d) of the ambient noises can be computed as [14]:

$$
N(f)=N_{t}(f)+N_{g}(t)+N_{w}(f)+N_{t h}(t)
$$

Where Nt $=$ Represent turbulence noise, $\mathrm{Ns}=$ Represent shipping noise, $\mathrm{Nw}=$ Represent wind noise, $\mathrm{Nth}=$ Represent thermal noise, $\mathrm{f}=$ Signal frequency $\mathrm{s}=$ Represent shipping activity factor (ranges in between 0 and 1 ) $\mathrm{w}=$ Represent wind velocity $(\mathrm{m} / \mathrm{s})$

\section{Doppler Spread}

Doppler spread also affects acoustic channel and it results in performance degradation of networks in transmission and reception [1]. Considering all above differences, the protocols devised for TWSNs (ground based) are not feasible in UASNs in terms of underwater environmental conditions and communication medium which causes special characteristics and challenges. Although different routing protocols for UASNs have been already proposed considering the unique characteristics of underwater environment, still routing schemes for selecting the optimal packet size in UASNs is not available readily. In this paper, we, therefore, propose an adaptive routing protocol, named Adaptive Packet Size Selection Based Routing Protocol (APSSR) for UASNs. APSSR determines the optimal packet size adaptively on the basis of varying distances between sensor nodes and estimated BER. The rest of the paper is organized as follows. In Section 2 , related works are reviewed and motivation behind our work is discussed. The proposed protocol, APSSR, is described in Section 3. The performance evaluation results are shown in Section 4. Finally, Section 5 concludes the paper.

\section{RELATED WORK AND MOTIVATION}

In this section, different existing UASNs routing protocols are presented. We divide these protocols into two different categories. The first category is the localization-free routing protocol and the second one is the localization-based routing protocol.

\subsection{Localization-Free Routing Protocols}

This category of routing protocols does not require any location information of sensor nodes. This scheme relies on local information of nodes (i.e. depth, physical distance \& dynamic addresses) [15]. Sink nodes are deployed on the surface of water. The nodes near the surface will have low depth as compared to the nodes below the surface of water. Some existing locations free UASN routing protocols are described below.

This category of routing protocols does not require any location information of sensor nodes. This scheme relies on local information of nodes (i.e. depth, physical distance and dynamic addresses) [15]. Sink nodes are deployed on the surface of water. The nodes near the surface will have low depth as compared to the nodes below the surface of water. Some existing location free UASNs routing protocols are described as follows.

In [16], Depth Based Routing Protocol (DBR) for UASNs is proposed. DBR is the first routing protocol that doesn't rely on location information of nodes. DBR forwards the data packet to its neighbor nodes in broadcasting manner. Upon receiving the data packet, each receiving node compares its depth with the depth of sending node included in data packet. If a receiver node's depth is less than sender node's one, it will forward the data packet. Otherwise, it will discard it. While the protocol ensures efficient data delivery, the drawbacks are redundant transmissions of packets. It doesn't handle void region problem and also doesn't considers energy of nodes.

In [17], Hop-by-Hop Dynamic Addressing Based Routing (H2DAB) is proposed. H2DAB works on the basis of distance from sink location. If a node has some packet to send, it will send Request to Send (RTS) packet to its neighbor nodes and as the response on the RTS packet, neighbor nodes send Clear to Send (CTS) packets. The sender will select the node as next hop which has smallest cost towards the sink. This approach has advantages like no need to develop any routing tables as well as no location information is required. It uses multiple sinks by which congestion and packet loss problem are minimized. However, use of RTS and CTS packets will create delays and consume more energy. In [18], Diagonal and vertical Routing protocol (DVRP) has been proposed for UASNs that increase data delivery ratio. However, the drawback of this protocol is that nodes periodically send HELLO packets to discover neighbor nodes which is an overhead.

In [19], a Greedy and Depth Based Multi-Hop Routing Protocol (DMBR) is proposed that only uses single node to transmit data packet towards destination. While this protocol saves energy and enhances network lifetime, the major drawback of this protocol is that it doesn't handle the network void problems. In [20], Energy-Efficient Depth-Based Routing Protocol (EEDBR) for UASNs is proposed. The major objective of this protocol is to balance nodes energy and enhance network's lifetime. A sender node selects only that one hop neighbor nodes which are rich in energy and have lower depth value. The advantages of this protocol are that, at first, it uses multi-link structure, so that energy consumption 
of nodes closer to a sink node could be minimized. Secondly, it uses depth and energy information in routing process.

Thirdly, it handles mobility of nodes by exchanging information of depth and residual energy after certain interval. Limitation of this protocol is that due to continuous mobility of nodes the knowledge acquisition phase repeats after certain interval which creates high overhead.

In [21], an Energy Efficient Routing Protocol (ERP2R) is proposed which uses physical distance and residual energy of node to determine whether to select it as a next hop node or not. Every node maintains the lists of neighbor nodes which are at less distance from sink node and have more residual energy. When a node wants to relay a data packet, it will select the best nodes from the list and broadcast packet towards them. This protocol minimizes the chances of data loss through control flooding. At the same time, it increases the network lifetime by selecting only those nodes which are near to sink and have more residual energy. No mechanism for void zone handling is discussed. Furthermore, in order to achieve reliable link quality towards forwarder node(s), a node is also considered by using link quality estimator called Expected Transmission Count (ETX). Each forwarder node computes holding time (HT) before forwarding data in order to reduce the unnecessary flooding of data packets. HT is computed on the basis of node's residual energy, so that HT of node with high residual energy is always less. As a limitation, void zone problem is not addressed which is the main problem in greedy communication. Moreover, ETX link quality estimator uses number of transmissions for accurate results due to nodes mobility hence leads to an extra overhead.

In [23], Distance Based Reliable and Energy Efficient Routing Protocol (DREE) for UASNs is proposed. DREE provides energy balancing between nodes. In DREE Fuzzy link quality estimator is used for link quality measurement towards eligible forwarder nodes. Node selection is based on three metrics (i.e. high residual energy, high link quality and less distance towards sink) is considered as best relay node. DREE increases network lifetime and packet throughput ratio. Furthermore, fuzzy link quality estimator includes four other metrics (i.e. Smoothed Packet Received Ratio (SPRR), Asymmetric Link (ASL), Stability Factor (SF) and Channel Quality as an average SNR (ASNR). In order to measure accurate link quality these four metrics are to be computed through a number of HELLO packets. However, due to highly dynamic nature of UASNs, link quality changes as the time goes by, thus computation of link quality leads to extra overhead.

Prediction assisted single copy routing protocol (PASR) [24] uses aggressive chronological projected graph (ACPG) algorithm to predicts nodes mobility. In ACPG there are two stages; firstly the connections between nodes at different time slots re-transformed into edges and secondly route reservation phase in which best connection are selected as route. Every node maintains a prediction vector and continuously updates it in each time slot. On the basis of guidance provided by ACPG, PASR uses historical information and predicts future node contacts. No control flooding is used in this protocol and node chooses best next hop node to forward its packet. This mechanism increases network lifetime by reducing energy consumption. Only one sink is used which reduces network stability. Limitations of this protocol is that each node must execute ACPG algorithm which is an overhead. In addition, this protocol assumes that network operates in slots of time interval $\mathrm{T}$, therefore, it is difficult to agree all nodes on a single time. The reason is that each node broadcasts a HELLO packet at the start of each slot to show its existence and exchange necessary information which generates additional overhead in the network.

In [25], A Hydraulic Pressure Routing (HydroCast) for UASNs is proposed to handle the void zone problem and to enhance the network reliability. In HydroCast, routing decision is made just like a depth-based routing protocol, where only low depth nodes are considered as eligible forwarder among the neighbor nodes and data packet is forwarded greedily towards that node having lowest depth. Furthermore, the HydroCast uses two modes; one is greedy mode and second one is void recovery mode that solves the problem of local maximum (void zone) that existed in depth-based routing. HydroCast increases data delivery ratio with minimum end-to-end delay. However, the limitation is that no energy balancing mechanism is provided.

\subsection{Localization-Based Routing Protocols}

This category of routing protocols assumes that each node knows its geographical location and location of sink node. These protocols utilize the location information of nodes in order to make routing decisions. But due high frequency absorption problem in water, GPS is not applicable. This is the main limitation of these protocols. Some existing location based UWSN routing protocols are described.

In [26] a localization-based Protocol named as Vector Based Forwarding (VBF) Protocol is proposed. In VBF a straight and single virtual pipe (vector) is computed by source node towards sink node and data is forwarded through relay nodes that falls within this vector radius, these nodes are considered the only eligible Forwarders. This restriction leads to limitation of the VBF because in sparse network VBF faces the void zone problem due to single vector and greedy approach, even when a node exists right outside the virtual pipe. Also there is no mechanism for energy balancing is provided. Furthermore, finding location of nodes is very difficult in UASN due to inapplicability of GPS system. In [27] another localization-based Hop-by-Hop Vector Based Forwarding (HH-VBF) Protocol is proposed. In HH-VBF a vector is computed in greedy manner (hop-by-hop) in order to improve network performance. In $\mathrm{HH}-\mathrm{VBF}$ each sender node computes its own vector to create towards the destination 
node instead of computing a single vector as in VBF. Due to this scheme HH-VBF enhance the delivery ratio. Hop-by-hop VBF is considered as the successor of Vector Based Forwarding Protocol. Limitation is same like VBF i.e. void zone problem and finding location of nodes is very difficult in UASN due to inapplicability of GPS system. In [28] DFR (Directional Flooding Based Routing Protocol) for UASN is proposed which uses control flooding. This protocol uses assumption that all nodes know their geographical location and the location of their neighbor nodes also. In this protocol the node having data packet to forward calculates its current angle to the destination and reference angle of the previous sender. DFR solved void region problem by making required for each flooding zone to include one forwarder node. It also improves performance in terms of packet delivery through controlled flooding. Limitation of this protocol is that it assumes that all nodes know about their location and also location of one hop neighbors but in case of totally changing environment keeping location information is difficult. Another protocol (Focused beam routing) FBR [29] also assumes that all nodes knows their own location and also the location of sink. FBR uses different transmission power levels to reduce energy consumption. If a node has packet to send RTS packet with certain power level that contains source and destination information after receiving RTS each node will calculate its position with respect to source and destination and reply with CTS if it lies within angle. In this way node closer to sink will be selected as next relay node. RTS and CTS exchanges occur in FBR protocol which might result in extra delays and energy consumption due to redundant retransmissions if sender node doesn't find next hop relay node. In case of sparse deployment, no node might be available that fulfill above mentioned requirements.

In [30], localization-based routing protocol called sector-based routing with destination location prediction (SBR-DLP) is proposed. SBR-DLP is coupled with capability of location prediction, in order to increase the packet delivery ratio. In SBR-DLP, the first assumption is that the sink is mobile, secondly beside that every node knows its own location information and the mobility plan of the mobile sink. In SBR-DLP, each source node broadcasts check_neighbor control packet. Upon receiving check_neighbor control packet, the neighbor nodes transmit a check_neighbor reply containing the sector information of the neighbor node. The process of sector information computation is take place on distance from vector, drawn between the source and the sink node. When node receive check_neighbor_reply control packets from its neighbor nodes, source node then on the basis of their sector numbers allots priorities to the neighbor nodes, and a node is selected as a next eligible forwarder node which have minimum distance from mobile sink. Advantage of SBR-DLP is that it increases packet delivery ratio and removes the problem of excessive involvement of multiple relay nodes in forwarding process. Secondly due to water currents it is possible that the scheduled movements of sink node can be altered.
In existing multi-hop routing protocols [16], [20], [21], [22], [23], [24], [26], fixed packet size is mostly used and transmitted on both reliable and un-reliable communication links by assuming that links sate is highly reliable and hence faces high bit corruption, unnecessary re-transmissions and low throughput. The reason is the continuous nodes mobility and harsh underwater environment.

Moreover, acoustic signals used for communication incur high error rate in UASNs. Due to such constraints distance among sensor nodes and BER varies time-to-time which has a direct impact on packet size. Selecting fixed sized larger packets faces high bit corruption where BER is high, while on the other hand fixed sized smaller packets increases traffic overhead in the network. To perform an effective communication and to achieve high throughput in UASNs, selection of optimal packet size is of utmost importance. Even though research on underwater acoustic communication have been significantly gaining popularity in recent years, however, still routing schemes for selecting the optimal packet size adaptively for effective routing in UASNs is not available

readily.

Therefore, in this paper, we propose an adaptive routing protocol APSSR for UASNs. APSSR does not use any link estimators for selecting adaptive packet size and select optimal packet size adaptively based on both varying distances between sensor nodes and BER and select the best relay node on the basis of BER and optimal packet size.
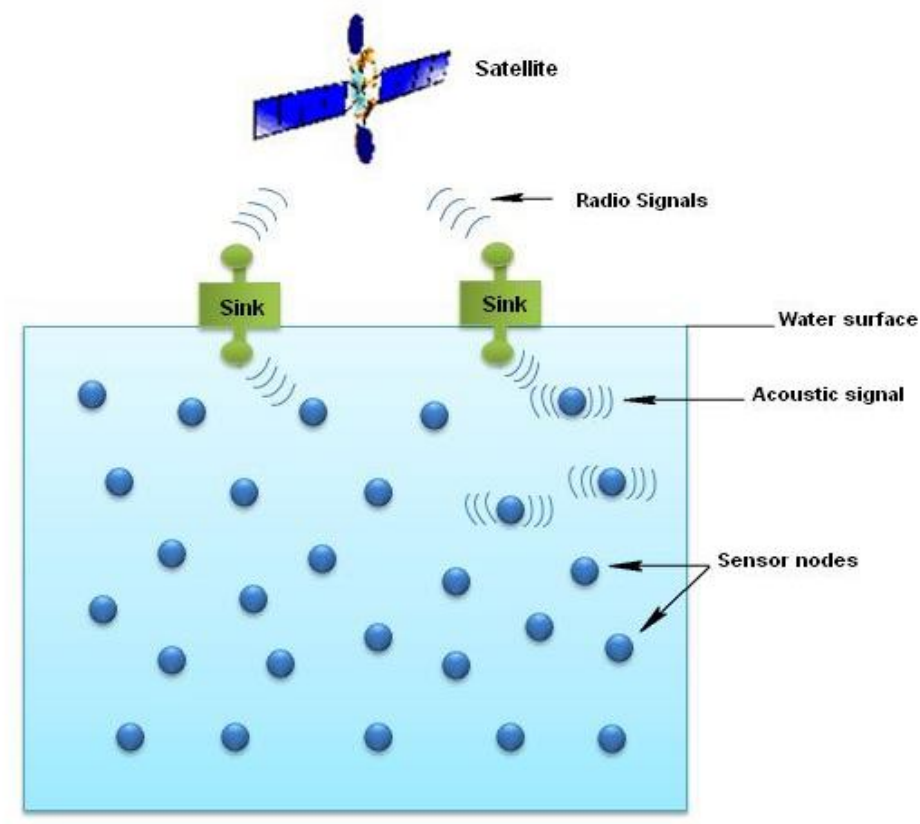

Figure 1: Underwater Acoustic Sensor Network

\section{PROPOSED ROUTING PROTOCOL: APSSR}

Figure 1 depicts an example of architecture of UASNs with multiple sinks which are deployed on the surface of water and equipped with both acoustic as well as radio modems. Sensor nodes 
placement is setup from top to bottom in the area of deployment. In our proposed protocol, there are two phases. The first phase is the knowledge acquisition phase and the second one is the optimal size packet forwarding phase.

\subsection{Knowledge Acquisition Phase}

In Knowledge Acquisition Phase, every node broadcast HELLO packet to its one hop neighbor nodes. Upon HELLO packets reception, nodes receiving the packets calculate the distance from its neighbor nodes and based on distance information, BER will be computed. The distance among sensor nodes towards their neighbor nodes is computed using Time of Arrival (TOA) method [4], [5]. Every source node then maintains its neighbor table containing distance and BER information about its eligible relay nodes (i.e. the nodes closer to the sink) as depicted in Figure 2. To compute the BER, over distance $\mathrm{d}$, we consider underwater channel model equation which is adopted from [25] and is defined as follows:

Distance between sender \& reciever $=\mathrm{d}=\mathrm{V}\left(\mathrm{t}_{1}-\mathrm{t}_{0}\right)$

$p e(d)=\frac{1}{2}\left(1-\sqrt{\frac{\Gamma(d)}{1+\Gamma(d)}}\right)$

$\Gamma(d)=\frac{\frac{\mathbb{E}_{b}}{A(d, f)}}{N_{0}}=\frac{E_{b}}{N_{0} l^{k_{a}}(f)^{d}}$

Where $\mathrm{d}$ represents distance (meter), $\mathrm{V}$ is sound velocity, to and $\mathrm{t} 1$ represent transmission and reception time of HELLO packet respectively, and pe(d) shows BER over distance $d$. In addition, $\Gamma(\mathrm{d})$ represents SNR over distance $d . A(d, f)=d k a(f) d$ shows the amount of path loss based on distance $d$ and frequency $\mathrm{f}$. Moreover, Eb is the average transmission energy per bit (joules), $\mathrm{N} 0$ is the noise spectral density (the noise power in a $1 \mathrm{~Hz}$ bandwidth $(\mathrm{dBm} / \mathrm{hz})$ ), and $\mathrm{k}$ is spreading factor. The geometry of propagation is described using the spreading factor $(1 \leq \mathrm{k} \leq 2)$ and $\mathrm{a}(\mathrm{f})$ is the absorption coefficient, described by the Thorp's formula in $(\mathrm{dB} / \mathrm{km})$.

The procedure of best relay node selection is given as follows.

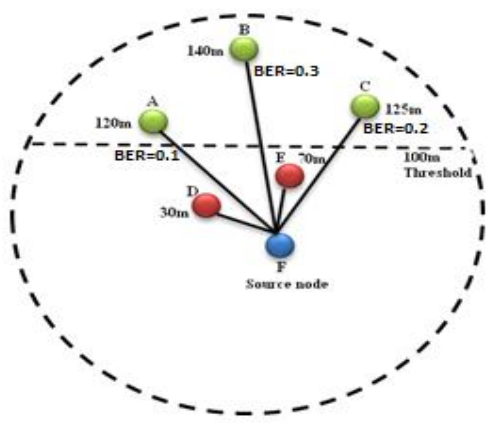

Figure 2: Best Next Hop Selection in APSSR
Figure 2 depicts the next hop selection process of our proposed APSSR protocol. Let's consider F is a source node and A, B, C, D and E are its neighbor nodes. Since the proposed protocol makes a decision for the next hop node based on distance and BER, a distance threshold is set to 100 meter. If neighbor nodes are less than 100 meter from the source node, the closer nodes will be selected as elgible forwarder that increases number of hops and may result in network delay. Based on this restriction, node $\mathrm{D}$ and $\mathrm{E}$ will not be selected as next hop nodes in forwarding decision. First of all, node $\mathrm{F}$ computes the distance towards $\mathrm{A}, \mathrm{B}$ and $\mathrm{C}$ (i.e. the nodes located above predefined distance threshold) and then on the basis of their distance information, BER will be computed for each neighbor node. A node with the lowest BER will be selected as an eligible forwarder node and its information (i.e. Distance and BER) will be stored in source node neighbor table at index "0". In Figure 2, since node A fulfills the required criteria of next forwarder node, therefore, node A will be selected as the best next relay node for forwarding. The Knowledge Acquisition Phase procedure is expressed in algorithm 1.

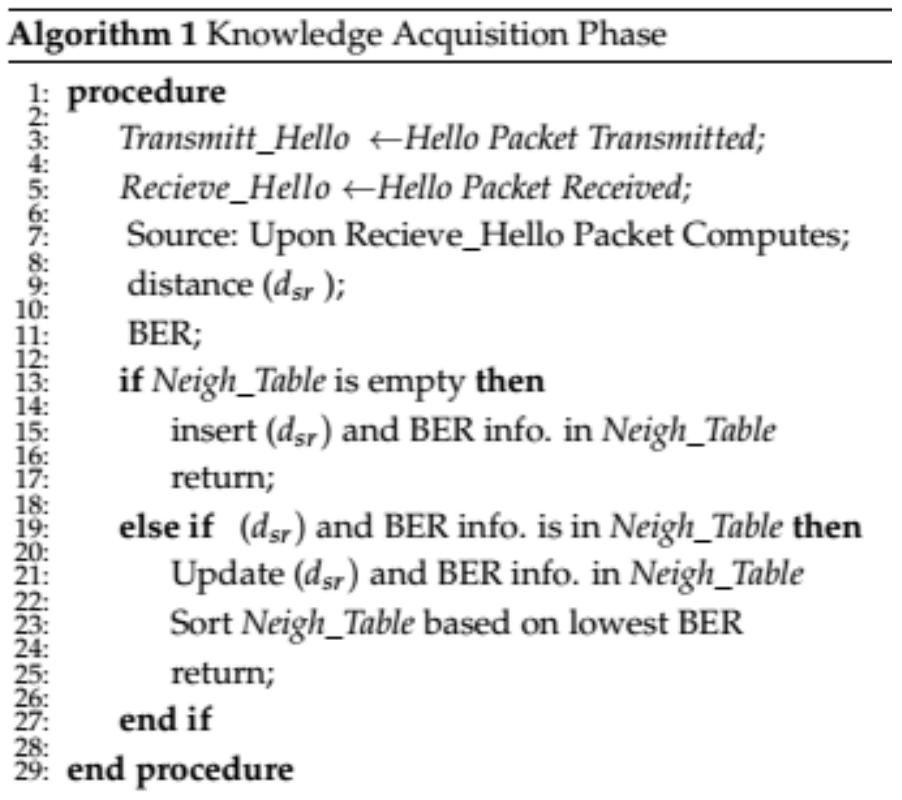

\subsection{Optimal Packet Size Forwarding Phase}

In this phase, when a source node has some data packets to send, it will not transmit the data packet immediately. First of all, it will perform the process to select optimal packet size adaptively on the basis of the best relay node information (i.e. distance and BER) and then this optimized packet size will be transmitted to the relay node. 
In APSSR, the optimal packet size can be determined using Equation (11) and is defined as follows:

$\mathbf{k}_{\text {opt }} \propto(1-p e(d))$

Where kopt represents optimal packet size and pe(d) represents BER over distance d.

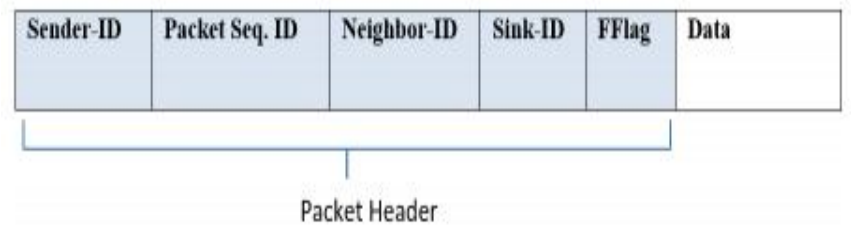

Figure 3: Format of the packet of APSSR

The format of the packet of the proposed protocol is shown in Figure 3. The header of packet consists of four fields: Sender-ID, Packet Sequence ID, Neighbor-ID, Sink-ID and a Fragmentation flag. The "Sender-ID" is the identifier of the source node, "Packet Sequence ID" is a unique sequence number (assigned by the source node to the packet), "Neighbor-ID" is the identifier of the best next forwarder node and the "Sink-ID" is the identifier of the destination node. The propose protocol utilizes the services of fragmentation and reassembling. In case next eligible forwarder node has high BER due to increase in distance among sender and receiver node, then the optimized packet size will be transmitted in the form of fragments (also adaptively computed) and will be reassembled at its selected best relay node. As depicted in Figure 3, there is a two-bit-length "fragmentation flag" field in the format which will identify that optimized packet size is transmitted in the form of fragments or not and it is represented by " 0 " and "1" (i.e. if optimized packet size is transmitted in fragments then the value of this field is set to " 1 " otherwise it will be set to " 0 ". This adaptive process will be continued until the complete data reaches to the sink node successfully. Figure 4 shows the complete process of optimal packet size forwarding in APSSR. In addition, if a node does not find any next hop above predefined distance threshold, then it will omit the threshold and select the next hop below the distance threshold value having lowest BER in order to reduce the chances of packet drop and delay. The process of optimal packet size computation and forwarding and optimal packet size reception is expressed in algorithm 2 and algorithm 3 respectively.

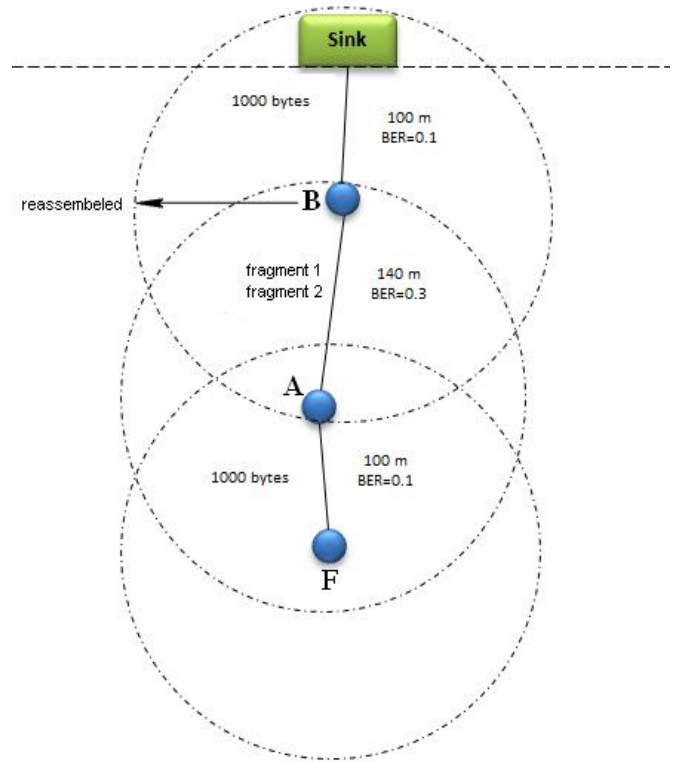

Figure 4: Process to forward the optimal size packets in APSSR.

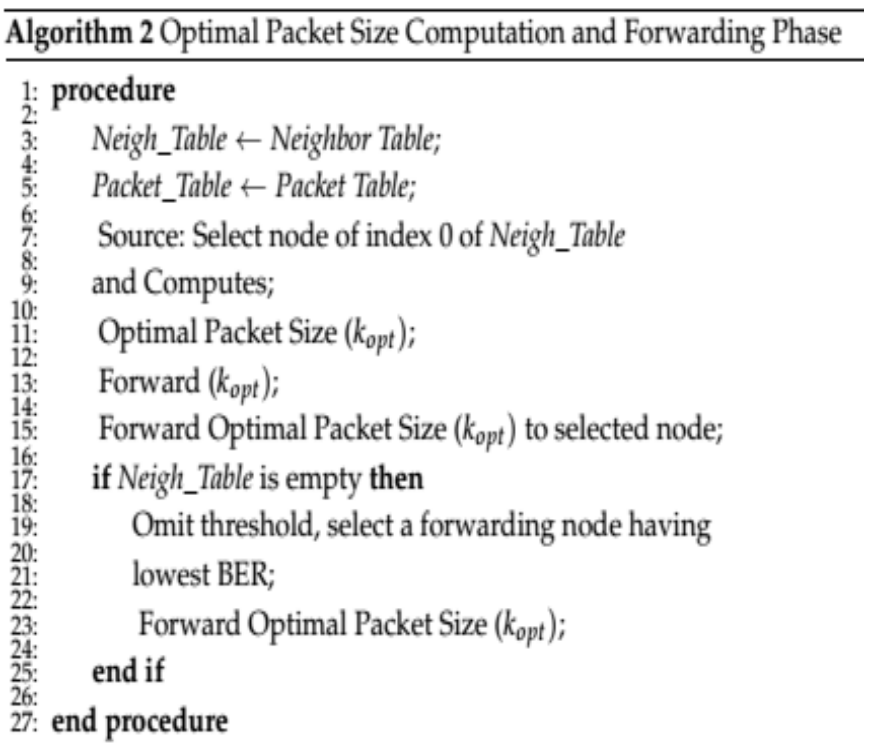




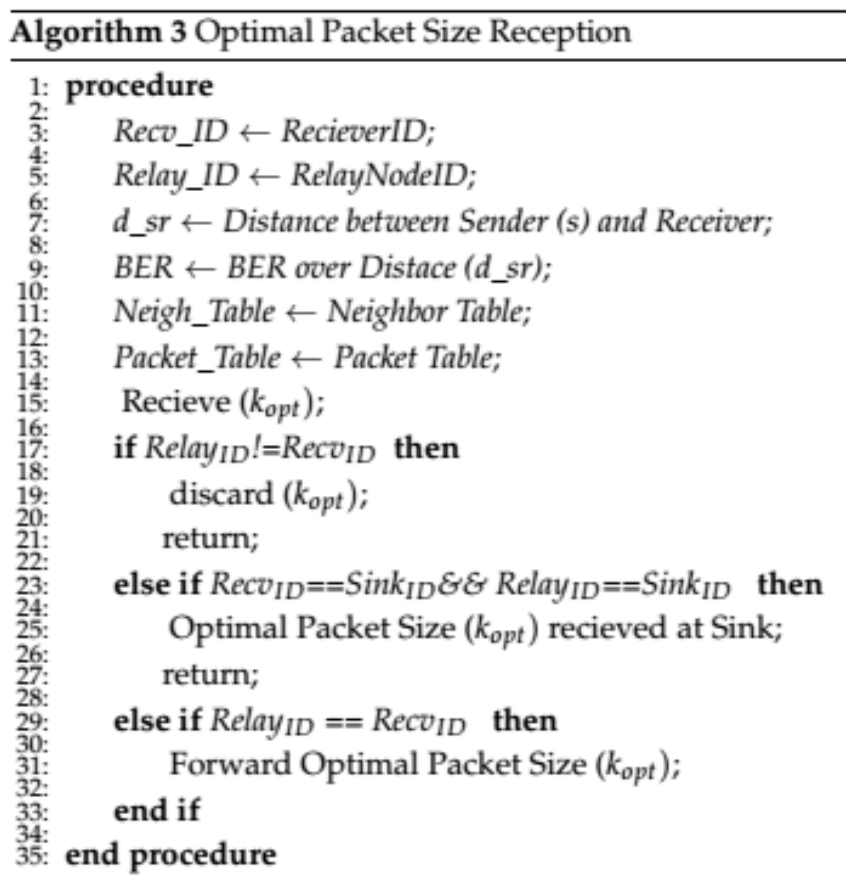

\section{PERFORMANCE EVALUATION}

\subsection{Simulation Environment}

This section shows the performance evaluation of APSSR using MATLAB simulator. In the simulation of 12000 rounds nodes have been randomly deployed. For performance evaluation we evaluate our proposed work by comparing it with existing well-known localization-free routing protocol DBR [16] and EEDBR [20]. We have used minimum data packet size of 1000 bytes. DBR and EEDBR both protocols transmit fixed data packet size. However, In APSSR optimal packet size is adaptively computed. Different simulation parameters are used and are summarized in Table 2.

Table 2: Simulation Parameters

\begin{tabular}{ll}
\hline Parameter & Value \\
\hline Simulator & MATLAB \\
Benchmark Protocol & DBR [16] and EEDBR [20] \\
Number of Nodes & $50,100,150,200$ \\
Bandwidth & $30000 \mathrm{~Hz}$ \\
Frequency & $30 \mathrm{kHz}$ \\
Transmission Range & $100 \mathrm{~m}$ \\
Transmission Power & $2 \mathrm{Watt}(\mathrm{W})$ \\
Receiving Power & 0.1 Watt $(\mathrm{W})$ \\
Receiving Power & $10 \mathrm{milliwatt}(\mathrm{mW})$ \\
Initial Energy & $70 \mathrm{Joules}$ \\
Minimum Data Packet Size & $1000 \mathrm{Bytes}$ \\
HELLO Packet Size & 48 Bytes \\
Area (m $\times$ m $\times$ m) & $200 \mathrm{~m} \times 200 \mathrm{~m} \times 200 \mathrm{~m}$ \\
Number of Rounds & 12000 \\
\hline
\end{tabular}

\subsubsection{Performance Metrics}

Following performance metrics are used to evaluate the performance of our proposed routing protocol. Network Lifetime: Network Lifetime is defined as the time when first node in network dies due to energy depletion. Data Received at Sink: Represent total amount of received data (bytes) at Sink node out of total amount of data send. Average Delay: Average delay is the total average time taken by the data packet to reach to destination node from source node.

Packet Reception Ratio: Packet Reception Ratio (PRR) is defined as the number of total packets per round received at Sink node. Packet Drop Ratio: Packet Drop Ratio is the number of packets dropped per round.

\subsection{Results and Analysis}

Figure 5 represents the network lifetime as a function of number of nodes between APSSR, DBR and EEDBR. DBR selects only those nodes having smaller depth as next hop relay. The energy of these smaller depth nodes rapidly exhausted and they die very soon. In addition, there are redundant packets transmission problem in DBR which leads to extra energy consumption. However, in EEDBR next hop is selected on the basis of depth and residual energy and only single node is allowed to transmit data packet. In EEDBR, no parameter has been taken into account to define a shortest and efficient path towards sink node. A node may forward data to node which is far away from sender and may result in higher energy consumption. In APSSR, next relay node is selected on the basis of distance and BER information, alternatively APSSR take into account efficient path selection parameter towards sink node. Due to mobility of nodes and acoustic signal usage for communications in UASNs distance among sensor nodes and BER changes frequently, therefore, same node is not selected every time in our proposed protocol. This alternatively achieved balance in energy consumption thereby, results in the prolong network lifetime. Furthermore, APSSR selects single node to transmit optimal packets size instead of flooding the data packet by each node, which also improves network lifetime.

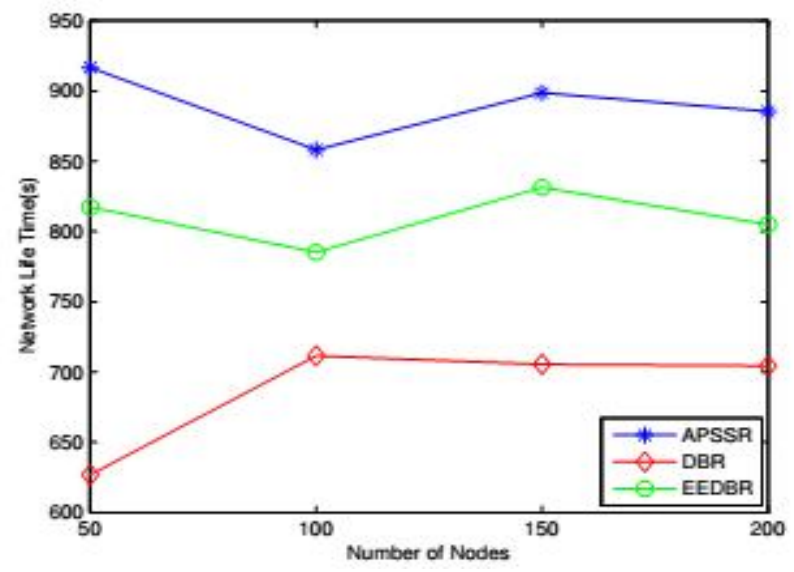

Figure 5: Network lifetime as a function of number of nodes 
Figure 6 depicts the total amount of data (bytes) received at sink node as a function of number of nodes between APSSR, DBR and EEDBR. In DBR and EEDBR total amount of data bytes received at sink node are lower than APSSR. The main reason is the fixed packet size transmission and not considering the quality of acoustic links, due to which bit corruption rate is relatively high as compare to APSSR. However, in our proposed protocol optimal size packet is selected and transmitted adaptively based on both varying distances among sensor nodes and BER, due to which bit corruption ratio is much lower and data successfully received ratio at destination is usually higher than DBR and EEDBR.

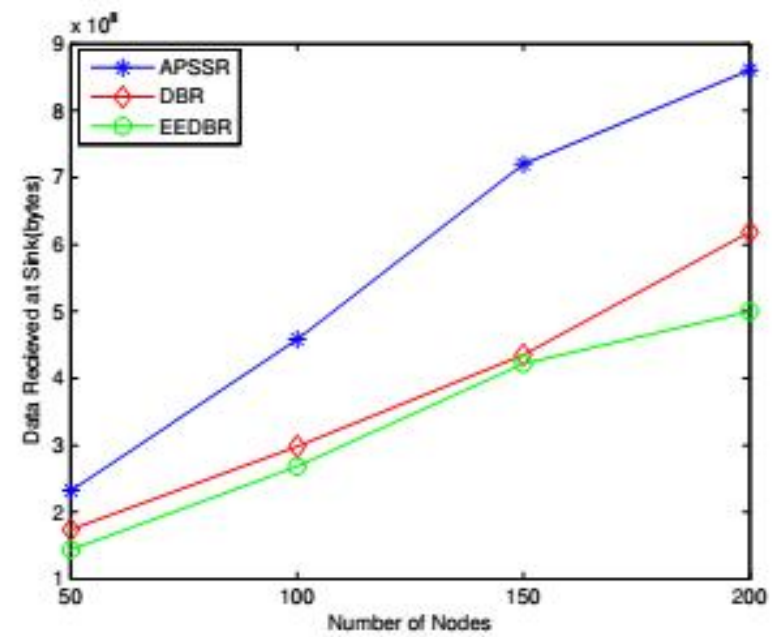

Figure 6: Data received at sink node as a function of number of nodes.

Figure 7 shows average delay comparison between APSSR, DBR and EEDBR as a function of number of nodes. It is noticed that with the increase in network density delay in DBR continuously increasing because the number of broadcasting nodes and holding time of the packet also increases. In DBR every node calculates holding time when it receives data packet. When holding time of node expires, it transmits the data packet. In contrast, delay in APSSR and EEDBR is almost similar and is relatively low as compared to DBR. The main reason is that there is no holding time in APSSR and EEDBR. Source node forwards the data packets as soon as it receives it, without any wait by selecting the single best next hop in neighbor list of forwarder nodes. Therefore, delay is reduced in both APSSR and EEDBR as compared to DBR.

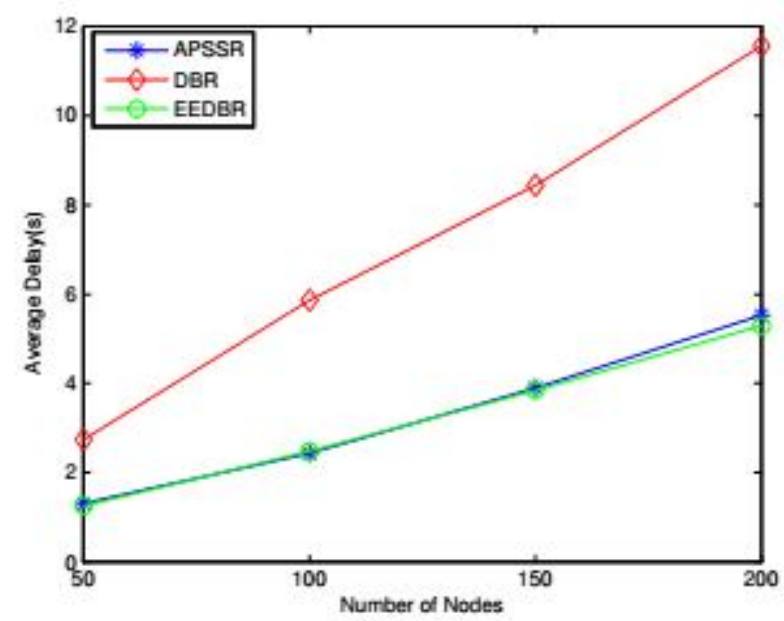

Figure 7: Average delay as a function of number of nodes

Figure 8 shows the throughput during the network lifetime in terms of Packet Reception Ratio. DBR and EEDBR shows less packet reception ratio than APSSR. The reason is the inadequate packet size transmission and ignoring the high BER on paths due to the highly dynamic nature of UASNs. While APSSR takes into account the distance among sensor nodes and BER based adaptive criterion as mentioned earlier for optimal packet size selection and next best hop selection, which greatly improves the receiving capability of packets at the destination. Another reason is that nodes in APSSR are alive for long time as compared to DBR and EEDBR, therefore, throughput in APSSR is relatively higher than DBR and EEDBR.

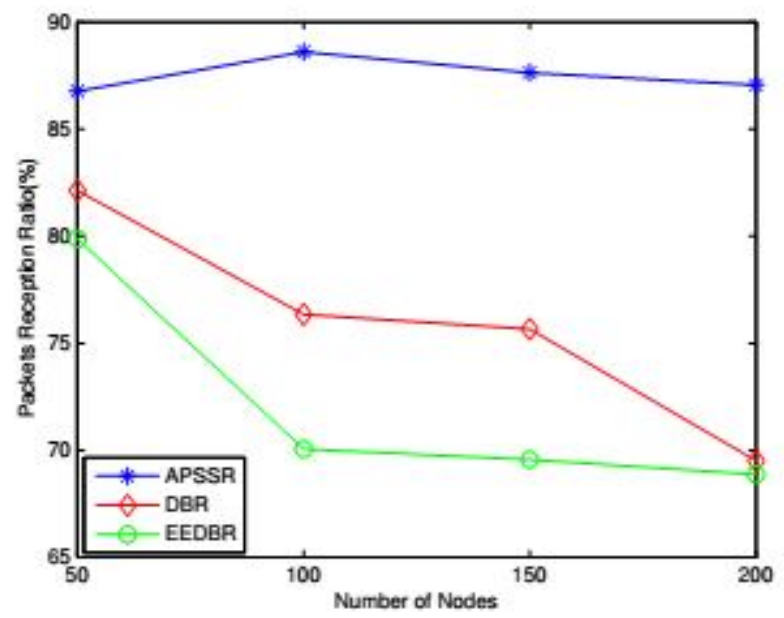

Figure 8: Packet reception ratio as a function of number of nodes

Figure 9 depicts the comparison of packets drop ratio between APSSR, DBR and EEDBR by increasing the number of nodes. APSSR selects optimal packet size adaptively by considering the selected next hop path length (distance) and path condition (BER) which greatly reduced the high bit corruption. APSSR selects optimal packet size adaptively by judging the path condition. Furthermore, APSSR uses fragmentation and reassembling services for such situations where bit corruption rate is high. Therefore, packet drop ratio 
is very low than DBR and EEDBR. However, in DBR and EEDBR fixed size packets are transmitted without considering the efficient path selection towards sink node, which leads to high bit corruption since acoustic links are highly error prone due to use of low-speed acoustic signals. Therefore, packet drop ratio is low in APSSR as compared to DBR and EEDBR.

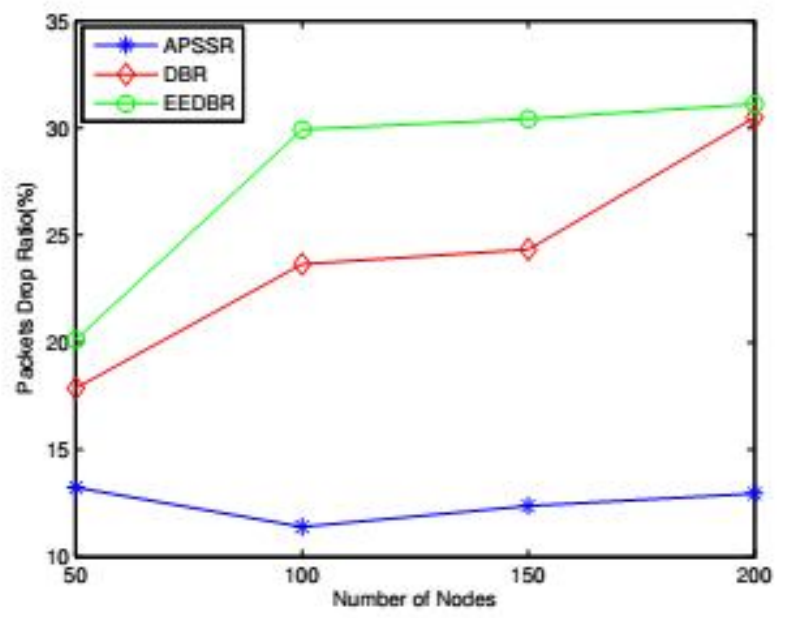

Figure 9: Packets drop ratio as a function of number of nodes.

\section{CONCLUSION}

In this paper we proposed an adaptive routing protocol APSSR for UASNs that determines the optimal packet size adaptively based on varying distances between sensor nodes and estimated BER. The proposed scheme has two phases i.e. Knowledge Acquisition phase and Optimal Packet Size forwarding phase. In Knowledge Acquisition phase, each node exchange small HELLO packets with its eligible neighbor nodes. Upon receiving HELLO reply to packets receiver node calculates the distance among its eligible neighbor nodes and based on distance information BER is computed and then each node maintains this information in its neighbor table priority wise. In Optimal Packet Size forwarding phase, forwarder node selects the optimal size packet adaptively based on both distance and BER information of selected next hop. Then selected optimal packet size is transmitted to that best next hop having lowest BER.

APSSR is compared with well-known underwater routing Protocol DBR [16] and EEDBR [20]. Simulation results show better performance in terms of Network lifetime, Data Reception Ratio at sink node, Network Delay, Packet Reception Ratio and Packets Drop Ratio. It is observed that data packet size is an essential parameter whose appropriate selection has a significant impact on overall network performance. This research investigated that how a wrong selection of the packet size can result in a high cost to network performance. We determined that the optimal packet size intensely relies on the varying distances between sensor nodes and BER. Therefore, network designers should select the appropriate packet size based on the path length between sensor nodes and channel condition of acoustic environment.

As a future work we will compare our proposed protocol with other underwater routing protocols by including link stability parameter.

\section{REFERENCES}

1. Kheirabadi, M. T. and Mohamad, M. M., (2013), "Greedy routing in underwater acoustic sensor networks: A survey," Int. J. Distrib. Sens. Networks, vol. 2013.

2. Sozer, E. M., Stojanovic, M. and Proakis, J. G., (2000), "Underwater Acoustic Networks," IEEE Journal of Oceanic Engineering, vol. 25, pp. 72-83.

3. Akyildiz, I. F., Pompili, D. and Melodia, T., (2005), "Underwater Acoustic Sensor Networks: Research Challenges," Ad Hoc Networks Journal (Elsevier), vol. 3, pp. 257-281.

4. Chandrasekhar, V. and Seah, W. K. G., (2006), "Localization in Underwater Sensor Networks - Survey and Challenges," pp. 33-40.

5. John Heidemann, Y. L., Ye, W., Wills, J. and Syed, A., (2006), "Research Challenges and Applications for Underwater Sensor Networking," proc. WCNC 2006, Las Vegas, NV, USA.

6. Ayaz, M., Baig, I., Abdullah, A. and Faye, I., (2011), “A survey on routing techniques in underwater wireless sensor networks,” J. Netw. Comput. Appl., vol. 34, no. 6, pp. 1908-1927.

7. Wang Ping, D. X., Dong-hao, F., Chang-qing, Z., Jian-chun, X., Qi-liang, Y., (2013), "A Reliable and Efficient Routing Protocol for Underwater Acoustic Sensor Networks," Int. J. Distrib. Sens. Networks, pp. $1-12$.

8. Cui, J.-H., Kong, J., Gerla, M. and Zhou, S., (2006), "Challenges: Building Scalable Mobile Underwater Wireless Sensor Networks for Aquatic Applications," IEEE Network, Special Issue on Wireless Sensor Networking, vol. 20, pp. 12-18.

9. Domingo, M. C., (2008), "Energy analysis of routing protocols for underwater wireless sensor networks," vol. 31, pp. 1227-1238.

10. Coutinho, R. W. L., Boukerche, A., Vieira, L. F. M. and Loureiro, A. A. F., (2016), "Design Guidelines for Opportunistic Routing in Underwater Networks," no. February, pp. 40-48.

11. Protocols, R., (2016), "A Survey on Underwater Acoustic Sensor Network Routing Protocols".

12. Darehshoorzadeh, A. and Boukerche, A., (2015), "Underwater Sensor Networks: A New Challenge for Opportunistic Routing Protocols," no. November, pp. 98-107.

13. Saraswathi, K., Netravathi, K. A. and Ravishankar, S., (2014), "A Study on channel modeling of underwater acoustic communication," vol. 3, no. 1, pp. 143-147. 
14. Sty and distance in an underwater acoustic communitojanovic, M., (2007), "On the relationship between capacication channel," ACM SIGMOBILE Mobile Computing and Communications Review, vol. 11, pp. 34-43.

15. Partan, J., Kurose, J. and Levine, B. N., (2006), "A survey of practical issues in underwater networks," in Proceedings of ACM WUWNet'06, (Los angeles, CA, USA).

16. Yan, H., Shi, Z. J. and Cui, J. H., (2008), "DBR: Depth-based routing for underwater sensor networks," Lect. Notes Comput. Sci. (including Subser. Lect. Notes Artif. Intell. Lect. Notes Bioinformatics), vol. 4982 LNCS, pp. 72-86.

17. Ayaz, M., Abdullah, A., Faye, I. and Batira, Y., (2012), "An efficient Dynamic Addressing based routing protocol for Underwater Wireless Sensor Networks," Comput. Commun., vol. 35, no. 4, pp. 475-486.

18. Ali, T., Jung, L. T. and Faye, I., (2014), "Diagonal and Vertical Routing Protocol for Underwater Wireless Sensor Network," Procedia - Soc. Behav. Sci., vol. 129, pp. 372-379.

19. Guangzhong, L. and Zhibin, L., (2010), "Depth-Based Multi-hop Routing protocol for Underwater Sensor Network," 2010 2nd Int. Conf. Ind. Mechatronics Autom., pp. 268-270.

20. Wahid, A. and Kim, D., (2012), “An energy efficient localization-free routing protocol for underwater wireless sensor networks," Int. J. Distrib. Sens. Networks, vol. 2012.

21. Wahid, A., Lee, S. and Kim, D., (2011), "An Energy-Efficient Routing Protocol for UASN using Physical Distance and Residual Energy," pp. 1-6.

22. Wahid, A., Lee, S. and Kim, D., (2012), "A reliable and energy $\square$ efficient routing protocol for underwater wireless sensor networks," International Journal of Communication System.

23. Tariq, M., ShafieAbd Latiff, M., Ayaz, M., Coulibaly, Y. and Al-Areqi, N., (2015), "Distance based Reliable and Energy Efficient (DREE) Routing Protocol for Underwater Acoustic Sensor Networks," J. Networks, vol. 10, no. 5, pp. 311-321.

24. Guo, Z., Wang, B. and Cui, J., (2010), "Prediction Assisted Single-copy Routing in Underwater Delay Tolerant Networks," pp. 1-6.

25. Lee, U., Wang P., Noh, Y., Vieira, L. F. M., Gerla, M. and Cui, J.- H., (2010), "Pressure routing for underwater sensor networks," in Proceedings of the IEEE INFOCOM, pp. 1-9.

26. Xie, P., Cui, J.-H. and Lao, L., (2006), "VBF: Vector-Based Forwarding Protocol for Underwater Sensor Networks," in Proceedings of IFIP Networking'06, (Coimbra, Portugal), pp. 228-235.

27. Nicolaou, N., See, A., Xie, P., Cui, J. H. and Maggiorini, D., (2007), "Improving the robustness of location-based routing for underwater sensor networks," in Proceedings of the Mts/Ieee Kobe-Techno-Ocean (OCEANS '07), pp. $1-6$.

28. Dongseung Shin, D. K., Hwang, D., (2011), "DFR: an efficient directional flooding-based routing protocol in underwater sensor networks," no. January 2011, pp. 1517-1527.

29. Jornet, J. M., Stojanovic, M. and Zorzi, M., (2008), "Focused beam routing protocol for underwater acoustic networks," Proc. third ACM Int. Work. Underw. Networks, pp. 75-82.

30. Wang Ping, D. X., Dong-hao, F., Chang-qing, Z., Jian-chun, X., Qi-liang, Y., (2013), "A Reliable and Efficient Routing Protocol for Underwater Acoustic Sensor Networks," Int. J. Distrib. Sens. Networks, pp. $1-12$.

31. Research paper I., (2009), "Sector-based Routing with Destination Location Prediction for Underwater Mobile Networks". 